\title{
Design of Bio-nanosystems for Oral Delivery of Functional Compounds
}

\author{
Miguel A. Cerqueira • Ana C. Pinheiro • Hélder D. Silva • Philippe E. Ramos • \\ Maria A. Azevedo • María L. Flores-López - Melissa C. Rivera • \\ Ana I. Bourbon · Óscar L. Ramos • António A. Vicente
}

Received: 19 July 2013/Accepted: 13 November 2013/Published online: 1 December 2013

(C) Springer Science+Business Media New York 2013

\begin{abstract}
Nanotechnology has been referred to as one of the most interesting topics in food technology due to the potentialities of its use by food industry. This calls for studying the behavior of nanosystems as carriers of biological and functional compounds aiming at their utilization for delivery, controlled release and protection of such compounds during food processing and oral ingestion. This review highlights the principles of design and production of bio-nanosystems for oral delivery and their behavior within the human gastrointestinal (GI) tract, while providing an insight into the application of reverse engineering approach to the design of those bio-nanosystems. Nanocapsules, nanohydrogels, lipid-based and multilayer nanosystems are discussed (in terms of their main ingredients, production techniques, predominant forces and properties) and some examples of possible food applications are given. Phenomena occurring in in vitro digestion models are presented, mainly using examples related to the utilization of lipid-based nanosystems and their physicochemical behavior throughout the GI tract. Furthermore, it is shown how a reverse engineering approach, through two main steps, can be used to design bio-nanosystems for food applications, and finally a last section is presented to discuss future trends and consumer perception on food nanotechnology.
\end{abstract}

M. A. Cerqueira - A. C. Pinheiro · H. D. Silva

P. E. Ramos - M. A. Azevedo · M. L. Flores-López ·

M. C. Rivera - A. I. Bourbon · Ó. L. Ramos ·

A. A. Vicente $(\square)$

Institute for Biotechnology and Bioengineering (IBB), Centre of

Biological Engineering, University of Minho, Campus de

Gualtar, 4710-057 Braga, Portugal

e-mail: avicente@deb.uminho.pt
Keywords Nanostructures - Bioactive compounds . Nanotechnology $\cdot$ Food

\section{Introduction}

Nanotechnology is a field that involves manufacturing, processing and application of structures, devices and systems by controlling shape and size at the nanometer scale. Recently, there has been a great interest in the study of the behavior of nanosystems as carriers of biological and functional compounds and in the understanding, control and utilization of those systems for functions such as delivery, control release and protection for food and pharmaceutical applications [1, 41]. Applications in the food industry are focused on the development of nanosized delivery systems for functional ingredients and additives (e.g., vitamins, antimicrobials, antioxidants, flavorings, colorants and preservatives), and innovative food packaging (e.g., incorporation of nanofibers and/or engineered nanoparticles) [16, 119]. Due to their sub-cellular size, nanosystems can improve solubility, bioavailability and sensorial aspects (e.g., mask flavors), prevent undesirable chemical reactions, protect functional compounds against chemical degradation and control the release of functional compounds, especially those with poor solubility in aqueous matrices. This behavior is not only related to the large surface area-to-volume ratio typically found in such nanosystems, but also to the influence of physical and chemical interactions between materials at the nanoscale, which have a significant effect on the overall properties of those systems. Size reduction in materials introduces, e.g., a great improvement in bio-adhesive properties that includes an increase in adhesive force and prolonged gastrointestinal transit time, and a large surface contact area 
per volume, leading to a higher bioavailability, when compared with larger particles [1]. Based on these unique characteristics, nanosystems can solve some problems occurring when using systems at macro- and micro-scale for delivery of functional compounds; such problems are: compatibility (e.g., aggregation and phase separation) with the food matrix (influencing, e.g., appearance, texture, stability or flavor of the product); release, that should be controlled and only activated once inside the human gut (i.e., some compounds start to be released when mixed with the food product and functional compounds lose their activity) and loss of activity of some functional compounds, affected by light, oxygen and temperature when dispersed in the food matrix [70, 119].

One of the greatest challenges when using nanosystems for food applications is the replacement of non-food-grade materials by bio-based, biodegradable food-grade alternatives. Polysaccharides (e.g., alginate, pectin, dextran and chitosan), proteins (e.g., zein, whey protein isolate) and lipids (e.g., medium chain triglycerides, tristearin and corn oil) are some of the logical options to address that challenge, as they present distinct advantages of biodegradability and lack of toxicity while also opening the door to new functionalities and applications. Nanosystems can be classified based on [66, 103]:

- the major material used in their fabrication;

- the production method (e.g., bottom-up or top-down);

- the predominant forces in the system (e.g., electrostatic, hydrogen bonding);

- the main properties of the system (e.g., mechanical and optical properties) and

- the system's overall free energy (thermodynamic or kinetic stable systems).

One of the major trends in the development of nanosystems is to combine different approaches such as mixtures of the materials used, combination of bottom-up and top-down strategies and intervention of different types of forces during the production process [19, 39, 47, 122] in order to achieve a desired functionality. In the last years, a large number of different delivery nanosystems have been developed, often using a trial-and-error approach, which leads to a great number of developed and well-characterized nanosystems, however, without a final and conclusive application. Based on this, the utilization of a reverse engineering approach (methodology where the required functionality of the final product is well known and therefore the whole developing process is tailored based on the desired properties/characteristics of the product), a very well-known approach in other fields but only marginally used in food science, could allow designing tailor-made bio-nanosystems [113] with a specific functionality. Equally, this approach should contribute to ensure that novel nanosystems are edible and that they can be pointed as usable by the food industry.

This review focuses on the design of bio-nanosystems for delivery of functional compounds, mainly on the main aspects of development, characterization and application of nanocapsules, nanohydrogels, lipid-based nanosystems and nanomultilayer systems in the size range between 10 and $300 \mathrm{~nm}$. The main aspects that should be considered when using these bio-nanosystems in the human gastrointestinal tract are also discussed. In addition, an opinion will be given on the reverse engineering approach applied to the design of bio-nanosystems, as well as on the future trends and consumer perception.

\section{Nanocapsules}

Nanocapsules, also called nanoparticles, are constituted by an external polymeric membrane and an internal part composed by a liquid or polymeric matrix that contains the active compound [27]. During nanocapsules' development, the selected method for their production is an important step because the structures must have properties allowing the performance of their functions properly and effectively. The method depends on the physicochemical character of the polymer, the loaded bioactive component, the final application and the desired properties for the nanocapsule (e.g., particle size, size distribution, surface area, shape, solubility, encapsulation efficiency and releasing mechanism) [26, 77, 86].

The methods for the preparation of nanocapsules are divided into three main techniques: polymerization, dispersion of preformed polymers and ionic pre-gelation/ coacervation [86, 87] (Fig. 1).

\section{Ionic Pre-gelation/Coacervation}

Ionic pre-gelation/coacervation methods use biodegradable hydrophilic polymers (e.g., chitosan, sodium alginate and gelatin) [64] and are based on the ability of polyelectrolytes to cross-link in the presence of a counter-ion to form nanocapsules (see Table 1), being the counter-ion responsible for cross-linking of ionic polymer and also for triggering pre-gelation [31, 78, 92]. Ionic pre-gelation/ coacervation involves the addition of a polymer, with positive or negative charge, and a cationic (e.g., calcium chloride) or polyanionic (e.g., sodium tripolyphosphate) counter-ion. Then, a second polymer is added that allows polyelectrolyte complexation and nanocapsule formation. The bioactive component is entrapped in the core of the first polymer added and stabilized after the addition of the second polymer. The polyelectrolyte solutions and bioactive component are added in a counter-ion solution drop 
Fig. 1 Methods for nanocapsules formation

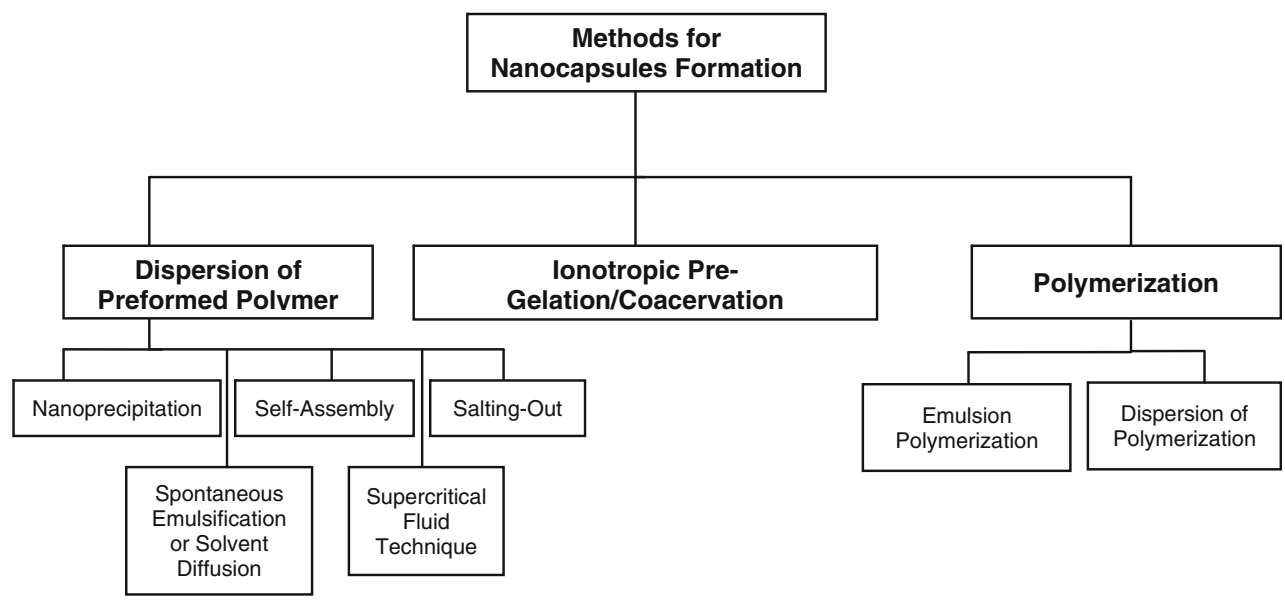

wise with a needle under magnetic stirring [14, 92]. This methodology is based on physical-chemical mechanisms, therefore, it is affected by several parameters such as stirring, flow rate of solutions, polymers characteristics (e.g., molar mass, flexibility and charge), $\mathrm{pH}$, ionic strength, concentration and polymers ratio [26]. The concentration of polymer $(0.05-1 \% \mathrm{w} / \mathrm{w})$ and of the counterion solution $(0.05-18 \mathrm{mM})$ has a direct influence on particles size, whereas the high concentrations produce biggersized nanocapsules [39, 117]. Molecular weight of the used biopolymers was also shown to influence the size of nanocapsules; $\mathrm{Hu}$ et al. [40] produced chitosan-tripolyphosphate nanoparticles and showed that the increase in molecular weight of chitosan from 150 to $300 \mathrm{kDa}$ leads to an increase in nanocapsules sizes from 173.3 to $309.7 \mathrm{~nm}$. In some cases, the high concentration of the counter-ion solution is responsible for creating a continuous gel that enables the production of nanocapsules. The polymer ratio has also an important influence in nanocapsules sizes [92]. Nanocapsules produced by this method can be used in food industry, for example, to encapsulate curcumin, tea catechins and capsaicin [21, 39, 117].

\section{Polymerization}

Several methods are used to prepare nanocapsules through polymerization of monomers using classical polymerization or polyreactions. Emulsion polymerization and dispersion polymerization are the two methods that use this chemical reaction process [86].

\section{Emulsion Polymerization}

This method involves the emulsification of hydrophobic polymers in water by an oil-in-water emulsifier. With polymerization, a large oil-water interface is generated and capsule nuclei are formed [18]. Surfactants or protective soluble polymers can be used to prevent nanocapsules aggregation, however, the type of surfactants can affect the size of nanocapsules, which is normally around $100 \mathrm{~nm}$ and when the bioactive compound is added to the polymerization medium, the encapsulation efficiency (i.e., relation between the amount of functional compounds encapsulated in the nanosystem and the total amount of functional compound initially added during the preparation) can be around $100 \%$ [86, 87]. The use or not of surfactants allows classifying this technique as conventional emulsion polymerization or surfactant-free emulsion polymerization, respectively. For conventional emulsion polymerization, it is also necessary to use water, a monomer with low water solubility (e.g., methyl methacrylate), a water-soluble initiator such as ammonium persulfate and a surfactant (e.g., sodium dodecyl sulfate). On the other hand, for surfactant-free emulsions, deionised water is also used, together with a water-soluble initiator such as potassium persulfate and monomers such as vinyl or acryl monomers. After the reaction, nanocapsules can be separated by centrifugation and/or filtration and then washed using water and organic solvents [76, 86, 124].

\section{Dispersion Polymerization}

It is defined as a technique of polymerization by precipitation, because a monomer is polymerized in the presence of a polymeric stabilizer soluble in the reaction medium. The reaction medium must be a good solvent for both the monomer and the steric stabilizer polymer. Thus, dispersion polymerization consists in having a homogeneous solution of monomer(s) with an initiator and a dispersant, being the nanocapsules formed by precipitation of the polymers [45]. 


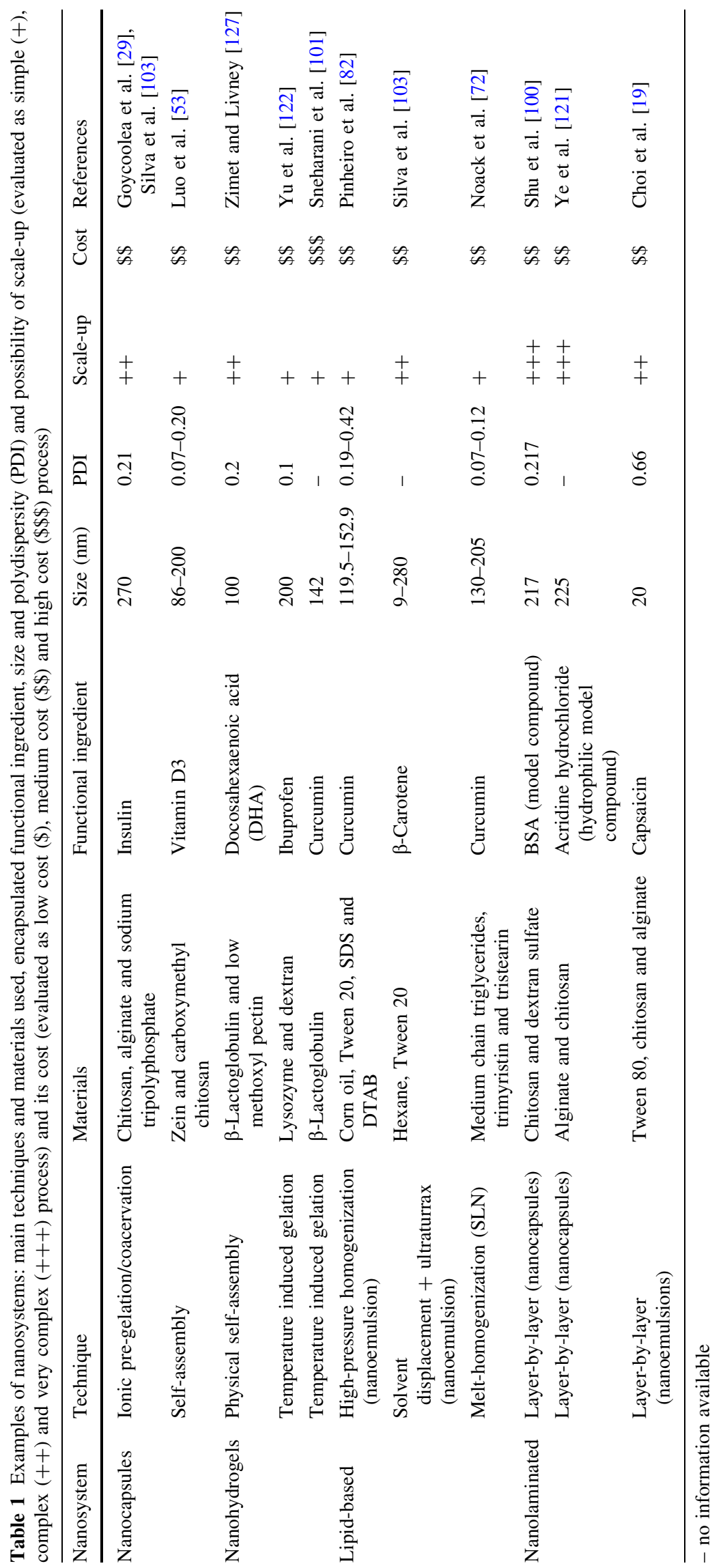


Dispersion of Preformed Polymers

In this case, nanocapsules are obtained, directly preformed, from synthetic, semi-synthetic or natural polymers [87]. The methods that are based on the preformed polymer technique are described hereafter.

\section{Nanoprecipitation Method}

This methodology is also called solvent displacement method; it uses three components: polymer, e.g., polylactic-co-glycolic acid (PLGA), polylactic acid (PLA), poly( $\varepsilon$ caprolactone) (PLC), poly(alkylcyanoacrylate (PACA) and poly( $\beta$-hydroxybutyrate) [87], solvent (organic phase) such as acetone, ethanol or hexane and non-solvent (aqueous phase) such as water $[6,86]$. Essentially, this method consists in the precipitation of a polymer, from an organic solution, and the diffusion of solvent in the non-solvent medium. The bioactive component for nanoencapsulation is dissolved in the solvent phase and a surfactant can be used. It is important to select appropriate molecules as solvent and non-solvent, because they can be different for each bioactive component. The size of nanocapsules obtained by nanoprecipitation is around $100 \mathrm{~nm}$ and they have been reported to exhibit a good stability, higher encapsulation efficiency $(>75 \%)$, sustained release, increased cellular uptake and bioavailability $[26,65,86]$.

\section{Spontaneous Emulsification or Solvent Diffusion}

This method involves the formation of a conventional oilin-water emulsion between a water-miscible solvent, containing a polymer and the bioactive component, and an aqueous phase, containing a stabilizer (surfactant). The rapid diffusion of the solvent from the internal to the external phase leads to polymer aggregation and formation of nanocapsules [84].

\section{Self-assembly}

This process uses polymers with capacity to form spontaneously compact and stable nanocapsules. The main driving forces for self-assembly are amphiphilicity and some weak interactions such as van der Waals, capillary, $\pi-\pi$ and hydrogen bonds. Thus, this method consists in a polymer structure organization without help or guidance from external agents [118]. The nanocapsules formed by selfassembly are dependent on the size and shape of polymer, composition of solution and environmental stresses. Materials such as zein, casein, chitosan and polylactic acid are the examples of polymers used in this method (see Table 1). Typically, nanocapsules have an average size of 50-100 nm [91]. Some works show that this process is able to produce nanocapsules that can be used in food industry, e.g., in the encapsulation of vitamin D3 [49].

\section{Salting-Out}

It is a modified method of emulsion that does not use organic solvents which can be hazardous to the environment as well as to physiological systems; in fact, nanocapsules are formed without surfactants or chlorinated solvents [86]. Salting-out implies the use of a polymer, an organic solvent totally miscible in water (e.g., acetone) and a salting-out agent (e.g., magnesium chloride, calcium chloride and magnesium acetate). The polymer and the bioactive component (lipophilic) are dissolved in an organic solvent and then a high concentration of salt is added, under vigorous stirring, for a strong salting-out effect in the aqueous phase. This process forms oil-in-water emulsions that are dissolved in water, causing the precipitation of the water-insoluble polymer.

\section{Supercritical Fluid (SCF)}

Some of the methods mentioned above use solvents or surfactants that are toxic. The use of SCF method appears as an alternative because these are environmental friendly solvents $[86,106]$. The main drawback associated is usually the high costs involved in SCF production.

The main disadvantages of nanocapsules production are related to some steps and materials used. The utilization of synthetic materials (non-food grade) in nanocapsules production (organic solvents, surfactants, monomers and initiators) [87] creates difficulties in their utilization by food industry. Also, the weak interactions (van der Waals, capillary, $\pi-\pi$ and hydrogen bonds) between materials during nanocapsules formation can also be a problem in nanocapsules production and their stability [118]. Another problem is the process of separation of nanocapsules; if not performed adequately, it can lead to nanocapsules' precipitation (being difficult to solubilize them after the separation), and can even destroy the nanocapsules therefore decreasing the final yield of intact nanocapsules.

\section{Nanohydrogels}

Innovative materials including smart polymer nanohydrogels are one of the central focuses of materials science due to their large surface area, available for, e.g., multivalent bioconjugation, and the availability of the interior network for the incorporation of bioactive compounds, thus possibly increasing their uptake, absorption and bioavailability [46, $50,110,115]$. The reduced size of nanohydrogels enables dispersion of water-insoluble additives (i.e., flavors, colors 
and preservatives), while increasing their stability and allowing a controlled release in food matrices.

Nanohydrogels are the three-dimensional hydrophilic polymer networks that can swell in water and hold a large amount of water while maintaining a network structure due to the presence of covalent bonds, hydrogen bonding, van der Waals interactions or physical cross-links [17, 33]. The water holding capacity and permeability are the most important characteristic features of a nanohydrogel. Their ability to absorb water is attributed to the presence of hydrophilic moieties such as hydroxyl and carboxyl groups as well as ethers, amines and sulfates in the polymers forming the nanohydrogel structure; this type of structures is responsible for the soft and elastic characteristic of such nanosystems [79].

The polar hydrophilic groups of nanohydrogels are the first to be hydrated upon contact with water, leading to the formation of primary bound water. As a result, the network swells and exposes the hydrophobic groups which are also capable of interacting with the water molecules. The network will absorb additional water, due to the osmotic driving force of the network chains toward infinite dilution. This additional swelling is opposed by the covalent or physical cross-links, leading to an elastic network retraction force. Thus, the nanohydrogel will reach an equilibrium swelling level.

Nanohydrogels are able to produce a pre-determined response to the alteration of certain environmental stimuli-e.g., temperature, $\mathrm{pH}$, light, electric or magnetic fields, ionic strength, solvent composition, redox potential or enzymatic conditions, at a desired point and time [28, 51, 98, 125]. These stimuli-sensitive nanohydrogels can display changes in the swelling behavior of the network structure according to external environmental conditions; they may exhibit positive thermo-sensitivity of swelling, in which polymers with upper critical solution temperature (UCST; temperature at which mixture of two liquids, immiscible at room temperature, ceases to separate into two phases) shrink by cooling below the UCST [89, 94].

Nanohydrogels can be produced using different techniques; however, the most commonly used is the gelation process [112]. Gelation is a phenomenon that typically includes the linking of polymeric chains, leading to a progressively larger embranchment of molecules, yet soluble polymers, depending on the network density, structure and conformation of the starting material. The aggregation of polydisperse soluble ramified polymers is called "sol." The continuous cross-linking increased the size of the ramified polymer chains decreasing its solubility. This continuous building process is called the "gelation" and results in polymers formation. The transition from the aggregation to a continuous building process is called "sol-gel transition" (or gelation) and the critical point

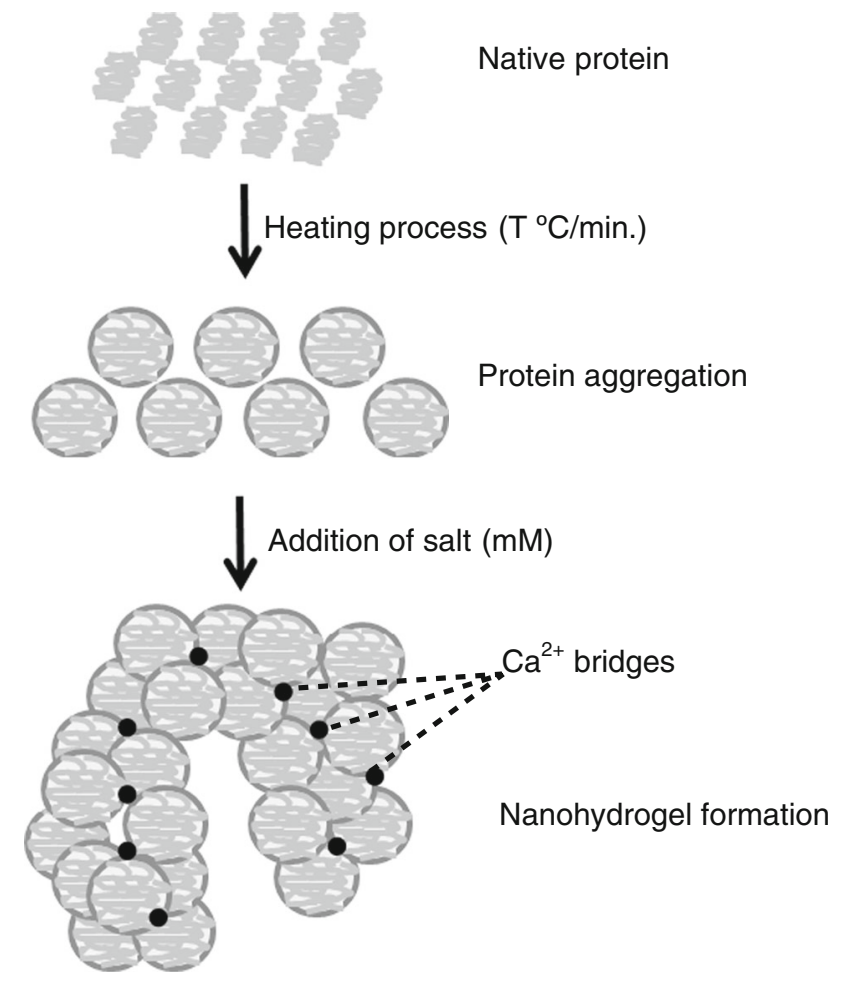

Fig. 2 Scheme of production of a protein nanohydrogel using a thermal and salt addition method to promote gelation

where gel first appears is called the "gel point" [88]. Figure 2 exemplifies the production scheme of a protein nanohydrogel using thermal and salt addition methods to promote gelation, which was the same procedure used by Maltais et al. [55].

Nanohydrogels can be produced either by physical or chemical gelation. Both forms present heterogeneous organization of independent domains, although they differ in the nature of molecular associations forming the network. Physical hydrogels are organized in heterogeneous clusters of distinct domains formed by molecular entanglements, free chain ends and molecular "hairpin," "kinks" or "loops" held together by weak hydrophobic associations, ionic interactions or hydrogen bonding [37]. Also called "reversible" or "pseudo" gels, physical nanohydrogels exhibit high water sensitivity (degrade and even disintegrate completely in water) and thermo-reversibility (melt to a polymer solution when exposed to heat). Because of the strong electrostatic interactions involved, $\mathrm{pH}$ is by far the most important and most widely used factor for controlling the strength and direction of physical hydrogels.

Chemical nanohydrogels (also called "irreversible" or "permanent" gels) are networks of polymer chains covalently linked at strategic connection sites. Most commonly, cross-linking is not spontaneous but is deliberately induced by reaction with small molecules such as aldehydes [36], 
radiation or UV light [43]. Uneven distribution of crosslinking within the gel leads to the development of some zones in which typical "reversible" features are still dominant and other zones with permanent properties arising from the cross-linked network. Chemical nanohydrogels neither disintegrate nor dissolve in aqueous solutions. Rather, they hydrate and swell until an equilibrium state is reached, which in turn strictly depends on the extent of the cross-linking.

Depending on several conditions (e.g., temperature, ionic strength, salt addition), that impact the unfolding of polypeptide chains and therefore the aggregation process, the gels formed can exhibit different microstructural properties, which are strongly related to the aggregates' molecular structure. For instance, during the thermal treatment, aggregation of molecules occurs and a balance between attractive and repulsive forces between unfolded molecules, either aggregates or gels, can exhibit various structures and morphologies. The ionic strength (positive and negative ions) can change the charge interactions that affect largely the aggregation process or even induce different conformational states. This may result in different aggregation behaviors and appearance of aggregates. The salt type is another condition that should not be neglected. Divalent cations (e.g., calcium, iron and magnesium) can promote aggregation in a different extent than monovalent cations (e.g., sodium) $[69,85]$. In this sense, depending on the aforementioned conditions, gelation can lead to clear or opaque gels. Opaque gels are formed by colloidal-sized "precipitates" that associate further to form particulate gel structures and are believed to contain fibrous structures, with diameters of the order of tens of nanometers [20].

Thermal nanohydrogels are produced by the unfolding of polypeptide chains with concomitant exposure of initially buried hydrophobic amino acid residues and subsequent self-aggregation of polymer molecules into a network that entraps water by capillary forces [17]. Forces involved in the aggregation process include hydrophobic effects, van der Waals, hydrogen bonding and covalent interactions [126]. The typical time-temperature combinations needed for nanohydrogels production range between 10 and $60 \mathrm{~min}$ and 50 and $80{ }^{\circ} \mathrm{C}$, which may limit their application to formulations that contain heat-sensitive ingredients; however, the heat treatments applied will depend on the biopolymer used, as well as on its concentration. Different polymers exhibit distinct intrinsic properties such as melting point or denaturation temperature. For instance, in the case of proteins, concentration and/or molecular weight will be the crucial parameters to determine the optimal time-temperature combination required to produce a stable nanohydrogel.

Due to the aforementioned attractive properties (i.e., forces and variable composition) of nanohydrogels, these nanosystems are potentially beneficial in biotechnology and, in particular, in the food industry. In the food industry, the use of bio-based materials to develop environmentsensitive nanohydrogels for bioactive compounds delivery constitutes an interesting strategy. A fundamental advantage of this approach is that functional carrier nanohydrogels can stabilize food texture, which is a highly desirable characteristic in the manufacturing of food products [74]. Due to their specific structure, size and composition, nanohydrogels exhibit a wide range of useful functionalities for the development of food products. For instance, viscoelastic properties play an important role allowing nanohydrogels to act as foaming and emulsifying agents, thus stabilizing food products. In addition, the molecular weight, shape and flexibility of biopolymers integrating nanohydrogels play also an important role [93]. Whey protein-based nanohydrogels are the good examples of the use of a food biopolymer in formulated foods due to its biological (e.g., digestibility, amino acid pattern, high biological value and sensory characteristics) and functional (e.g., emulsification, gelation and foaming) properties. Besides it is relatively inexpensive (by-product from the cheese industry), classified as GRAS (generally regarded as safe), it presents a high nutritional value as well as an extraordinary binding capacity to various active compounds [12, 23, 54].

Zimet and Livney [127] designed a stable proteinpolysaccharide nanohydrogels for encapsulation and delivery of hydrophobic nutraceuticals (e.g., DHA). These authors evaluated the potential use of $\beta$-lactoglobulinpectin nanohydrogels as nanocarriers for $\omega-3$ fatty acids (see Table 1). They observed that this nanocarrier entraps DHA molecules producing a stable system capable of protecting DHA against oxidation during an accelerated shelf-life stress test. Therefore, these nanohydrogels are able to protect nutraceutical compounds against deterioration, thus importing health properties to beverages and food products during storage. Also, Somchue et al. [105] used $\beta$ lactoglobulin and hen egg white protein for encapsulation of $\alpha$-tocopherol. In order to protect $\alpha$-tocopherol from release in the gastric condition, alginate was used to coat these encapsulated nanohydrogels. The authors observed that it was possible to protect and maintain the stability of this bioactive compound using a protein-based material. Bengoechea et al. [8] prepared food-grade bovine lactoferrin nanohydrogels by a simple thermal method. The protein nanohydrogels produced were resistant to subsequent changes in $\mathrm{pH}$ (from 3 to 11) and to salt addition (from 0 to $200 \mathrm{mM} \mathrm{NaCl}$ ), and can be useful as functional ingredients in food products.

The use of nanohydrogels in food applications may present some limitations to formulations that contain heatsensitive ingredients, especially when these nanosystems 
are produced by thermal gelation. In addition, biodegradable nanohydrogels produced by physical gelation contain labile bonds in the polymer backbone or in the cross-links that can be broken under physiological conditions either enzymatically or chemically, in most of the cases by hydrolysis [35, 37]. Therefore, the ingredients entrapped into nanohydrogels can be degraded or even disintegrated completely during production and/or storage. On the other hand, other aspect that should not be neglected is the cost associated with their production, as it will be mainly dependent on the biopolymer used and the preparation technique applied (see Table 1).

\section{Lipid-Based Nanosystems}

Lipids as carriers have the potential of providing endless opportunities in the area of drug delivery due to their properties, ability to enhance both gastrointestinal solubilization and oral bioavailability of bioactive compounds; their physiochemical diversity and biocompatibility also have made them very attractive candidates as carriers for oral formulations [15].

Delivery systems that use the lipophilic phase as the core of the nanosystem can be either in the solid state or liquid state, depending on the physical state of the lipid at room temperature [15]. In this way, there are a variety of delivery systems (nanoemulsions, multilayer nanoemulsions, solid lipid nanoparticles (SLNs), nanocapsules and nanostructured lipid carriers) that can be used to fortify food products $[13,58,103,120]$. The following text will address mostly nanoemulsions and SLNs.

Table 1 shows a list of lipid-based nanosystems that can have the applications in the food industry.

\section{Nanoemulsions}

The food industry deeply relies on the utilization of emulsions: soft drinks, milk, cream, salad dressings, soups, mayonnaise, sauces, dips, butter and margarine [32]. Nanoemulsions can be used to design and develop novel functional ingredients, improving water dispersibility, thermal stability, oral bioavailability, sensory attributes and physiological performance [107]. Once they can be produced with natural compounds, thus minimizing the impact on the organoleptic properties of food products, they are capable of preserving bioactive compounds in their active form during storage and improve bioavailability during the gastrointestinal passage, as well the solubility of the bioactive compounds [95, 97].

Nanoemulsions can be produced through high-energy methods (making use of devices that use very high mechanical energy inputs) and low-energy methods (requiring low energy for nanoemulsions production and mainly depend on the intrinsic physicochemical properties of surfactants and the oily phase) [41, 102]. Briefly, emulsifiers adsorb to the formed droplets and reduce the interfacial tension, facilitating the disruption. Emulsifiers form a protective layer around the droplets, protecting them from aggregating [32]. It is important to state that there is not a unique ideal emulsifier for use in food products, being the emulsifier dependent on different parameters (type and concentration of other ingredients, production steps, environmental conditions related with manufacture and storage and way of utilization) [32].

Qian et al. [83] produced nanoemulsions stabilized with $\beta$-lactoglobulin to encapsulate $\beta$-carotene and examined their protective effect toward this compound. These authors also studied the influence of temperature, $\mathrm{pH}$, ionic strength and emulsifier type on the physical and chemical stability of $\beta$-carotene nanoemulsions. The color loss in these nanoemulsions was higher for increased storage temperatures $\left(5-55^{\circ} \mathrm{C}\right)$ and was faster at lower $\mathrm{pH}$ values (3-8). The degradation of $\beta$-carotene was independent of the ionic strength $(0-500 \mathrm{mM}$ of $\mathrm{NaCl})$. Nanoemulsions produced with $\beta$-lactoglobulin were unstable to aggregation at $\mathrm{pH}$ values close to the isoelectric point of the protein ( $\mathrm{pH} 4$ and 5), above the ionic strength of $\mathrm{NaCl}(>200 \mathrm{mM}$ at $\mathrm{pH}$ 7) and a temperature of $55^{\circ} \mathrm{C}$. The authors determined that $\beta$-carotene degradation was significantly slower in nanoemulsions stabilized with $\beta$-lactoglobulin as compared to those stabilized with Tween 20 .

Sessa et al. [96] encapsulated resveratrol in food-grade nanoemulsions using the high-pressure technique and evaluated physicochemical stability under accelerated ageing and during simulated gastrointestinal digestion. The antioxidant activity was measured at different stages of the digestion using Caco-2 cells, measuring the residual activity of resveratrol inside the cells. These authors showed that all formulations exhibited excellent antioxidant activity on Caco- 2 cells, obtaining more than $80 \%$ of cellular antioxidant activity, being these results comparable to the ones obtained by resveratrol dissolved in dimethyl sulfoxide. These results suggest that nanoemulsions may improve the uptake of antioxidant compounds into the cells. The nanoemulsions produced with glycerol monooleate and soy lecithin presented the highest values of cellular antioxidant activity, indicating that these formulations had a better resveratrol entrapment in the lipid phase, reducing the interaction of resveratrol with oxidant reagents. This was attributed to the formation of reverse micelles in the presence of the most lipophilic emulsifier within lipid droplets stabilized by the most hydrophilic emulsifier tested.

Nevertheless nanoemulsions have some drawbacks such as instability to freeze-thawing and to passage through 
human gastrointestinal GI tract. Their stability can be improved through the layer-by-layer (LbL) deposition technique, where the deposition of a biopolymer can better protect the encapsulated bioactive compounds. Scale-up is also a problem once, e.g., the high-pressure homogenization technique is a costly process (see Table 1), and in the case of the solvent displacement technique an evaporation step is needed to eliminate solvents like hexane or acetone, thus rendering this technique limited for food applications.

\section{Solid Lipid Nanoparticles}

Solid lipid nanoparticles (SLN) can also be used by the food industry as a delivery system, being developed to encapsulate, protect and deliver lipophilic bioactive compounds [34, 95]. Several methods can be used to produce SLNs, typically high-pressure homogenization (hot process), sonication, micro-emulsion, solvent diffusion and solvent emulsification evaporation. High-pressure homogenization is the most used technique to produce SLNs, despite requiring much energy consumption [22, 71]. The liquid lipid phase and an aqueous surfactant solution are homogenized, using temperatures above the melting point of the lipids in order to produce oil-in-water nanoemulsions; the nanoemulsion is subsequently cooled to temperatures below the crystallization point of the lipids, leading to the formation of SLNs [34]. The use of solid lipids has shown to increase the control of the release profiles, due to the solid matrix and higher stability and protection of the incorporated bioactive compound against chemical reactions such as oxidation [34, 63, 95].

Sessa et al. [95] extracted polyphenols from grape marc and were able to encapsulate them in nanoemulsions (using sunflower oil) and SLNs (using palm oil). SLNs exhibited different trends of physical stability under accelerated ageing when compared to the nanoemulsions (which remained stable in time). The use of glycerol monooleate as lipophilic emulsifier leads to the formation of SLN with a $\mathrm{Z}$-average diameter at the micrometric range $(1.3 \mu \mathrm{m})$, probably due to the formation of aggregates of the solid fat droplets. Sessa et al. [95] confirms this hypothesis by the evolution of the Z-average diameter over storage time, for lower temperatures. The chemical degradation was also evaluated; the solid particles were less efficient than the nanoemulsions, showing a slight decrease in the absorbance peak after 14 days for one formulation, and a significant decrease after 3 days for the glycerol monooleate formulation, being in agreement with the observed physical instability (creaming). In this paper, the cellular antioxidant activity was also measured and all the formulations exhibited a cellular antioxidant activity significantly higher than the unencapsulated grape marc polyphenols. This can be explained due to the nanometric size (176 and $220 \mathrm{~nm})$, resulting in an increased transport through the Caco- 2 cell membrane.

Helgason et al. [34] studied the effect of surfactants in the coverage of SLNs. These authors showed that the addition of surfactant after the homogenization step and prior to crystallization of nanoemulsions could lead to the production of SLNs with different morphologies and crystal structures. Additional adsorption of surfactants after homogenization occurred in both solid and liquid particles, being mostly absorbed to SLNs. The use of insufficient concentrations of surfactant during the crystallization process may lead to aggregation and particle destabilization. This study evidences that the addition of surfactants after homogenization can offer a viable means to improve stability and to control crystal structure and morphology of SLNs. Authors conclude that Tween 20 may not be the best surfactant to stabilize SLNs (tripalmitin). Nevertheless, if SLNs are to be utilized by the food industry it is important to develop effective strategies to prevent their aggregation and further instability [34].

Negi et al. [71] produced SLNs using the hot selfnanoemulsification technique: a hot mixture of lipid, drug and surfactant/co-surfactant was added to hot water under gentle agitation, spontaneous formation of hot nanoemulsions took place, due to self-emulsifying ability of the mixture, and by using rapid cooling the authors were able to form SLNs. The melting behavior of the SLNs was evaluated using DSC, the physical mixture (oil phase, surfactant and co-surfactant) was analyzed, showing all characteristic melting peaks of each component. But for the unloaded SLNs, only two peaks were obtained, with a similar melting point peak of the oil phase. This indicates that the oil became more amorphous after SLNs formation. After drug encapsulation, a reduction in the melting point of the oil phase was observed and onset temperature indicates a reduction in the lipids crystallinity. The absence of the drug melting point peak indicates a reduction in crystallinity (confirmed by XRD analysis) of the drug and more amorphous conversion.

Berton-Carabin et al. [9] evaluated the distribution and chemical reactivity of small molecules with varying lipophilicities (lipophilic, amphiphilic and hydrophilic) by electron paramagnetic resonance (EPR). The lipophilic molecules in emulsion were distributed between the lipid and aqueous phases, but in the SLNs they were expelled from the oil droplet core to the aqueous phase upon crystallization of the oil phase. The amphiphilic molecules in the emulsions were distributed between the lipid phase and the interface, but in the SLNs they were also expelled from the droplets to the interface upon crystallization of the oil phase. The hydrophilic molecules were present exclusively in the aqueous phase in both emulsions and SLNs. The expulsion of lipophilic ingredients from oil droplets 
generally led to increased physical accessibility and reactivity with water-soluble components in the aqueous phase [9]. The amphiphilic molecules presented the highest chemical stability among the three studied molecules due to their amphiphilic character, which lead to their accumulation in a stable form either at the emulsion and SLN interfaces, regardless of the lipid physical state. This study also showed that the location and stability of the lipophilic molecules were dependent on the physical state of the lipid, being largely encapsulated in the lipid phase, but they can quickly move to the aqueous phase. The hydrophilic molecules were on the aqueous phase being very mobile and accessible to water-soluble reagents, thus having the highest reactivity and being independent of the physical state of the lipid [9].

\section{Nanolaminated Systems}

Since its introduction in $1991, \mathrm{LbL}$ assembly technique has become a widely used method for the preparation of different nanolaminated systems [116]. This technique is a simple, inexpensive and highly adaptable method in which oppositely charged layers of polyelectrolytes are adsorbed on core materials with controllable thickness and properties $[11,44]$. However, the main limitations regarding nanolaminated systems are related to their preparation method. The prerequisite for successful LbL coating is the presence of a minimal surface charge, which is one of the few disadvantages of the technique [24]. Indeed, the key issue of $\mathrm{LbL}$ assembly is the need for surface recharging at each adsorption step. The molecules used for assembly should have a sufficient number of charged groups to provide stable adsorption on an oppositely charged surface and non-compensated charges exposed to the exterior [65]. The presence of charge is not the only factor that may affect multilayer formation. Surface texture and coating elasticity could also affect the adhesion of the coating to the substrate [24]. However, the LbL technique presents significant advantages, such as the simplicity of its process and equipment, the use of water as the main solvent, the flexible application to objects with irregular shapes and sizes and the control over thickness, composition and structure of the nanolaminated coatings.

The versatility of the LbL technique allows the use of diverse templates (e.g., planar and colloidal substrates) [73], resulting in the formation of different multilayer nanostructured systems such as nanofilms, nanoemulsions and nanocapsules (Fig. 3). The following sections show examples of multilayered nanostructure systems produced using both colloidal and planar templates and their respective applications in the food sector.

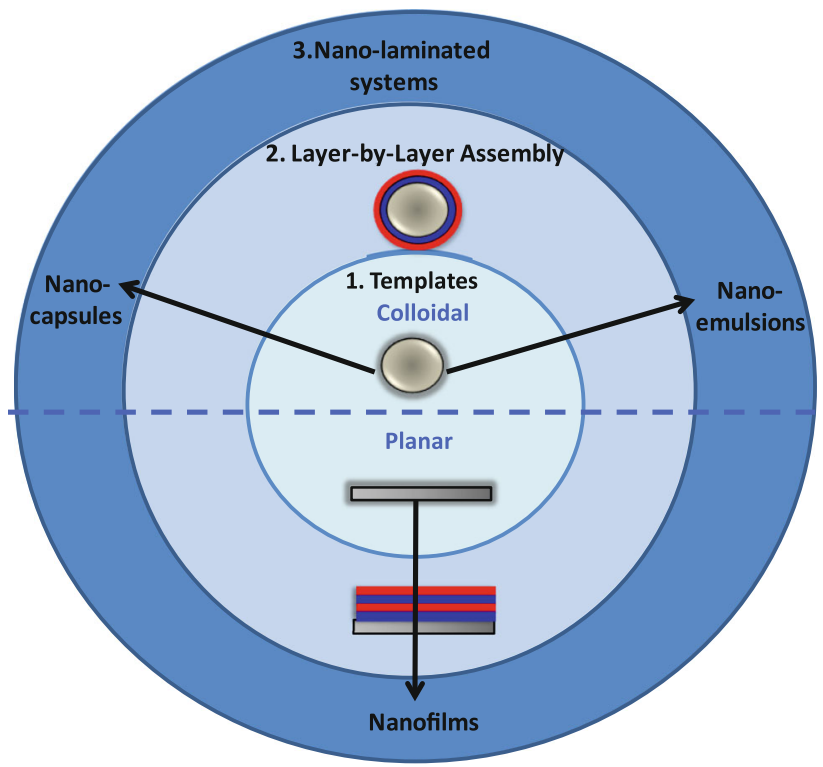

Fig. 3 Schematic illustration of nanolaminated systems prepared by layer-by-layer technique, using colloidal or planar templates. Adapted from Wang et al. [116]

\section{Planar Templates}

LbL deposition on planar templates involves the use of solid surfaces such as glass, quartz, polyethylene terephthalate (PET), silicon wafers, mica and gold-coated substrates [10]. These templates have been widely used to produce nanofilms. Nanofilms have potential applications in a wide range of areas including food packaging and they present significant advantages when compared with conventional edible films and coatings: they exhibit better physical stability in aggressive environmental situations and provide a better chemical stability to the incorporated active compounds and a greater control over the rate of release of these, because of the possibility of manipulating the thickness and the properties of the interfacial layer [59]. Also, they have the ability to incorporate functional agents (e.g., antioxidant, antimicrobial and nutritional additives) focused on extending shelf life and increasing food quality [62].

The effectiveness of functional nanofilms has been extensively evaluated and proved by recent works such as Mantilla et al. [56]. These authors validated the antimicrobial capacity of an alginate-based nanofilm system with a microencapsulated antimicrobial complex (beta-cyclodextrin and trans-cinnamaldehyde) applied in fresh-cut pineapples. The application of this nanofilm extended its shelf life to 15 days at $4{ }^{\circ} \mathrm{C}$ by inhibiting microbial growth. On the other hand, Pinheiro et al. [80] proved the efficacy of a $\kappa$-carrageenan/chitosan nanofilm on a PET support as a good gas barrier. In another work using the same system, Pinheiro et al. [81] contributed to clarify the loading and 
release mechanisms involved in structures at the nanoscale. These authors observed that the amount of the cationic compound methylene blue (MB) loaded into the system has increased with the distance from the first layer, suggesting that MB was able to diffuse into nanofilm and not only adhered to the surface of the layer immediately below it, and that the release of MB was due to both concentration gradient and polymer relaxation of the nanolayers.

\section{Colloidal Templates}

The most used colloidal templates include silica nanospheres [123] and polystyrene nanoparticles [121] for the production of multilayer hollow nanocapsules, and emulsion nanometer-sized templates for the preparation of multilayer nanoemulsions [7].

\section{Multilayer Hollow Nanocapsules}

Hollow nanocapsules can be obtained by dissolving the colloidal template using acid or solvent, after the LbL deposition procedure. This treatment is usually followed by multiple centrifugation-washing cycles, to assure complete removal of the core and to isolate the hollow nanocapsules [100]. These nanocapsules can be applied as delivery systems to carry, protect and deliver functional ingredients (e.g., drugs, antimicrobials, antioxidants, flavorings and colorants) to their specific site of action [99, 119]. The functional compounds can be embedded into the template (pre-loading) or loaded after nanocapsule formation (post-loading) [52].

Some examples of multilayer nanocapsules that can be applied in the food sector can be found in literature. Jamróz et al. [42] demonstrated the possibility of using a polysaccharide-protein complex (furcellaran/bovine serum albumin) as core for the alternated deposition of PDADMAC (polydiallyl dimethyl ammonium chloride) and PSS poly(sodium-4-styrene sulfonate) and obtained stable nanocapsules with sizes ranging from 60 to $80 \mathrm{~nm}$, that were considered very convenient, biocompatible carriers for biologically active molecules. Moreover, Yuxi et al. [123] have developed a multilayer hollow nanocapsule $(120-210 \mathrm{~nm})$ by the LbL deposition of two natural polysaccharides, Iota-carrageenan and chitosan. The authors concluded that this multilayer system might be a promising candidate as nanoreactor or nanocontainer to control the synthesis, encapsulation, and release behavior of bioactive compounds. Biodegradable hollow capsules were prepared through the LbL assembly of water-soluble chitosan and dextran sulfate on protein-entrapping amino-functionalized silica particles-see Table 1 [100]. This system demonstrated a good capacity for the encapsulation and loading of BSA and may offer a promising delivery system for watersoluble proteins and peptides.

\section{Multilayer Nanoemulsions}

The production of multilayer nanoemulsions involves the formation of multiple layers of emulsifiers and/or polyelectrolytes around the oil droplets in which the bioactive compound is incorporated. This approach consists in the adsorption of an ionic emulsifier to the surface of the oil droplets during homogenization to produce the "primary" emulsion, then, an oppositely charged polyelectrolyte is added and adsorbs to the droplet surface, forming the "secondary" emulsion; by repeating this procedure the nanoemulsions can be coated by three or more layers [57]. Multilayer nanoemulsions have been found to have better stability to environment stresses (e.g., pH, salt, thermal processing, chilling, freezing, dehydration and mechanical agitation) than conventional nanoemulsions with single layer interfaces [30, 111].

Different biopolymers (both proteins and polysaccharides) have been used to form the subsequent layers on top of the primary emulsions in order to provide the desirable properties to the emulsion droplets. Aoki et al. [4] have used a three-step process to produce nanoemulsions stabilized by sodium dodecyl sulfate (SDS)-chitosan-pectin membranes and showed that the tertiary emulsions had improved stability to environmental stresses. In a similar work, Hou et al. [38] produced stable emulsions incorporating $\beta$-carotene that were composed by oil droplets surrounded by soybean soluble polysaccharides and chitosan. These authors observed that the physicochemical stability of $\beta$-carotene emulsions has been improved by the adsorption of chitosan.

The LbL technique presents great potential for the generation of different multilayer systems with improved characteristics for functional compounds' delivery. Microscopy images of multilayer systems, i.e., nanofilms, nanocapsules and nanoemulsions can be found in Fig. 4 .

\section{Behavior of Bio-nanosystems in the Gastrointestinal Tract}

The knowledge of the behavior of bio-nanosystems as well as the fate of bioactive compounds encapsulated within them in the GI tract is of utmost importance for optimizing the bioactivity of such compounds and to ensure that these structures are safe for human consumption [60]. Also, it is possible to design delivery systems that release the bioactive compound to a specific site of action, such as mouth, stomach, small intestine, large intestine or colon [61]. For example, a nanolaminated coating can be designed so that its integrity or permeability changes in response to specific biological triggers (such as $\mathrm{pH}$, ionic strength or enzymes) [3]. 

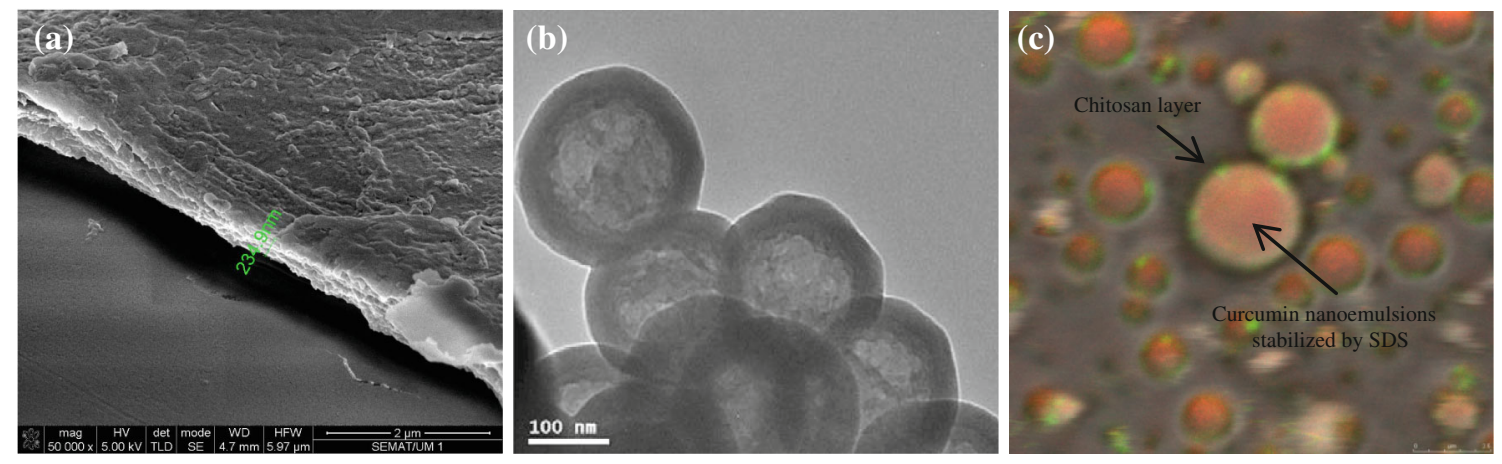

Fig. 4 Examples of multilayer nanosystems: a SEM images of the $\kappa-$ carrageenan/chitosan nanofilm with incorporation of MB (reprinted from Pinheiro et al. [81], copyright 2013, with permission from Elsevier). b TEM images of water-soluble chitosan/dextran sulfate

In recent years, different in vitro digestion models (mainly static models) have been used as a means for understanding the physicochemical behavior of bio-nanosystems within the GI tract. However, conversely to the extensive work focusing on the behavior of lipid-based delivery systems during in vitro digestion, few works can be found in literature about the behavior of nanocapsules, nanohydrogels or nanofilms within the GI tract. The behavior of nanoemulsions under simulated small intestinal conditions is commonly evaluated using the $\mathrm{pH}$-stat method, which is a simple in vitro lipolysis model [48].

The fate of nanoemulsions within the GI tract can be potentially altered by their initial characteristics, such as particle size, initial emulsifier and oil type, therefore the impact of those characteristics has been extensively studied in the last years. Salvia-Trujillo et al. [90] evaluated the influence of particle size on lipid digestion and on $\beta$-carotene bio-accessibility using emulsions with different initial droplet diameters. These authors observed that: (a) the rate and extent of lipid digestion increased with decreasing droplet diameter, which was attributed to the increase in lipid surface area exposed to lipase with decreasing droplet size and also (b) $\beta$-carotene bio-accessibility increased as the initial droplet size decreased, which was related to the fact that there was more undigested oil present for larger droplets, thus retaining $\beta$-carotene. Recently, Pinheiro et al. [82] produced curcumin nanoemulsions using three different emulsifiers: Tween 20 (non-ionic), sodium dodecyl sulfate (SDS, anionic) and dodecyltrimethylammonium bromide (DTAB, cationic)—see Table 1. A human gastric simulator was used as in vitro digestion model (in which the stomach, duodenum, jejunum and ileum steps were performed) to evaluate the impact of surface charge on the digestion of curcumin nanoemulsions. This dynamic model allowed the simulation of continuous peristaltic movements and consequently enabled a more mechanically realistic simulation of the dynamic digestion process. Those authors found that hollow nanocapsules incorporating BSA (reprinted from Shu et al. [100] copyright 2013, with permission from Elsevier). c Curcumin nanoemulsions stabilized by SDS and chitosan

emulsifier charge had a significant effect on lipid digestion and on curcumin bioavailability, probably because it alters the ability of bile salts and lipase to adsorb onto emulsion surfaces. The positively charged DTAB-stabilized emulsions were the least stable during the digestion process, exhibiting the largest increase in droplet size and eventual phase separation which probably contributed to the reported low curcumin bioavailability (Fig. 5).

The influence of droplet composition on lipid digestibility and curcumin bio-accessibility was examined by Ahmed et al. [2]. These authors found that long-chain triglycerides (LCT) were digested to a lesser extent than shortor medium-chain triglycerides (SCT or MCT), which was attributed to the fact that longer-chain fatty acids digestion products, contrary to shorter-chain fatty acids, present low water dispersibility and tend therefore to accumulate at the oil/water interface, preventing lipase from accessing the non-digested emulsified lipids. On the other hand, the bioaccessibility of curcumin was very low when SCT was used as the oil phase being the highest bio-accessibility obtained with MCT. The authors attributed these results to the fact that MCT and LCT can form mixed micelles capable of solubilizing curcumin, whereas SCT cannot.

One of the few works regarding the evaluation of the behavior of polymeric nanoparticles in the GI tract was recently developed by Arunkumar et al. [5]. In this work, lutein, a non-provitamin-A carotenoid, was encapsulated in nanoparticles composed of water-soluble low-molecularweight chitosan and lutein bioavailability was studied in vitro and in vivo, using lutein in mixed micelles as control. The bioavailability of lutein in nanoparticles was significantly higher $(27.7 \%)$ than control, and also the lutein level in plasma $(54.5 \%)$, liver $(53.9 \%)$ and eyes $(62.8 \%)$ of mice fed on nanoencapsulated lutein was higher compared to the control.

There is still a lack of knowledge regarding the biological fate of ingested bio-nanosystems and further 


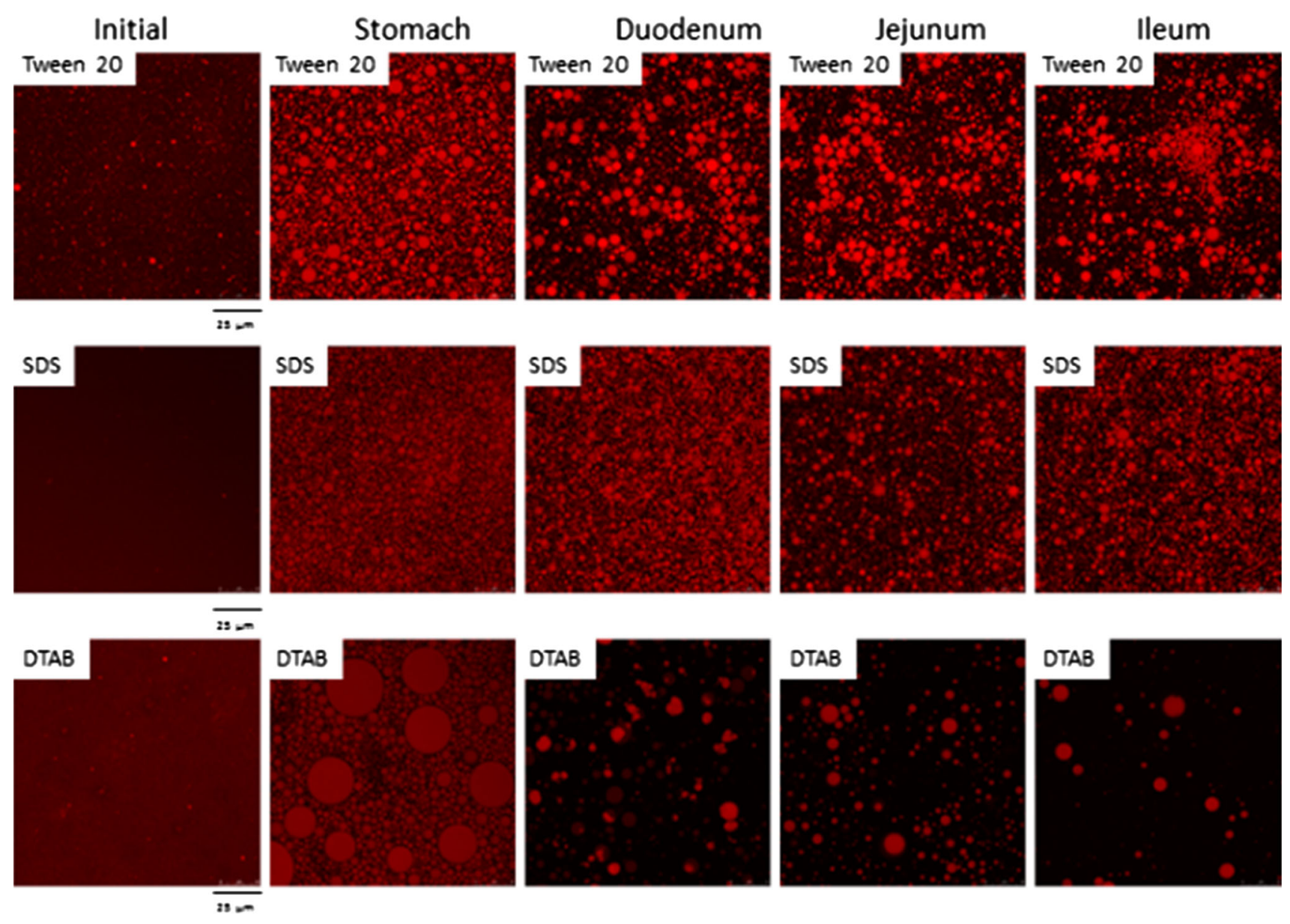

Fig. 5 Influence of emulsifier type on the morphology of curcumin nanoemulsions as they pass through an in vitro digestion model. The scale bar for all confocal images is $25 \mu \mathrm{m}$ [82]—reproduced by permission of The Royal Society of Chemistry)

research is needed to either access its safety and to produce tailored delivery systems (i.e., with optimized bioactivity). Also, being infeasible the use of in vivo models (high costs and ethical constrains often involved), there is a need of using more realistic in vitro gastrointestinal models, i.e., models that can accurately simulate the complex physicochemical and physiological processes that occur within the human gastrointestinal tract.

\section{Reverse Engineering Approach}

In the last years, a great number of works aiming at developing bio-nanosystems for functional compounds' delivery led to a significant accumulation of scientific knowledge concerning the structure of nanosized systems. However, studies where the structure-properties relationship for bio-nanosystems is established are still lacking.

Nowadays, the availability of sophisticated tools to analyze nanosized structures (e.g., dynamic light scattering, electron microscopy and X-ray scattering), the advanced knowledge of the desired functions of a system for functional compounds delivery (e.g., appearance, flavor, stability, bio-accessibility and bioavailability) and the availability of software that allows inter-relating the information gathered, shows that it is possible to create ondemand new bio-nanosystems providing specific functional performances. This knowledge can lead to a shift of the design paradigm of the bio-nanosystems, where a desired property/function can be tailored according to the delivery interest of a specific functional compound. This approach can be based in a reverse engineering approach where, e.g., the delivery of a functional compound is considered the central process and where a systematic study is performed backwards to find a feasible technology (i.e., materials and methods) to build a system with the required delivery properties.

There are several definitions of reverse engineering that change according to the field of application. Otto and Wood [75] referred reverse engineering as a process that initiates the redesign process, wherein a product is predicted, observed, disassembled, analyzed, tested, "experienced," 
and documented in terms of its functionality, form, physical principles, manufacturability and assemblability. Recently, Moskowitz and Maier [68] mentioned reverse engineering as a method by which the researcher begins with an objective to match, for example, a response or rating profile, and then searches among the alternative ingredient combinations to identify the specific combination that generates this profile, or comes as close as possible to this profile.

In a food science conception, the objectives of reverse engineering are to identify the variables that allow determining the ingredients that could have a required number of profile attributes (e.g., consumer acceptance and ingredient characteristics). Being so, the researcher has to identify the profile of ingredients' attributes that will match in the reverse engineering [67, 114].

The utilization of a reverse engineering approach to the design of bio-nanosystems appears to be a feasible option to cover some of the issues still pending related to the delivery/release of functional compounds. Designing a nanosystem for delivery of a functional compound should take into account several aspects that can be divided in two main features. The first feature is related with the desired function of the nanosystem, specifically where it will have to deliver and which type of functional compound will it carry; this information will allow understanding the compatibility between nanosystems and functional compounds in order to choose the most adequate ingredients for the subsequent development. Knowing where the functional compounds will be released is possible to know the kind of environments that the bio-nanosystems will be subject to before delivering. This information allows designing a nanosystem that could protect the functional compounds against mechanisms of chemical degradation (e.g., chemical degradation of lipophilic functional compounds in the stomach's acid medium); that could increase or at least avoid adversely affecting the bioavailability of the functional compound. At the same time, it is possible to understand whether the ingredients used for the nanosystems' development are compatible with the functional compound. This information will also allow having the perception of the ability of the bio-nanosystem to deliver the functional compound in a given site of action (i.e., delivery efficiency), which can be linked with the delivery mechanism.

The second feature is related with the materials used and the compatibility of the functional compound and the intended functionality of the nanosystems. Materials used should be food-grade ingredients and they should be processed using easily implemented operations; also, the ability of the nanosystem to retain the encapsulated material (i.e., a high loading efficiency of the functional compound) should be guaranteed.

Figure 6 shows the direction that should be taken during design and development of a bio-nanosystem for the delivery of functional compounds. Using a reverse engineering approach, the first step is to identify which is the desired functionality of the final bio-nanosystems (e.g., functional compound and site of action) and only then study the development conditions that will ultimately lead to the production of the bio-nanosystem (i.e., materials and methods selection). An example of application of this approach can be given with the delivery of water-soluble vitamins, such as vitamin B2, that presents a crucial role in the normal functioning of human brain and nervous system. The starting point is the knowledge about the needs that the system will have to cover: this vitamin should be absorbed
Fig. 6 Design of a bionanosystem for the delivery of functional compounds using reverse engineering approach

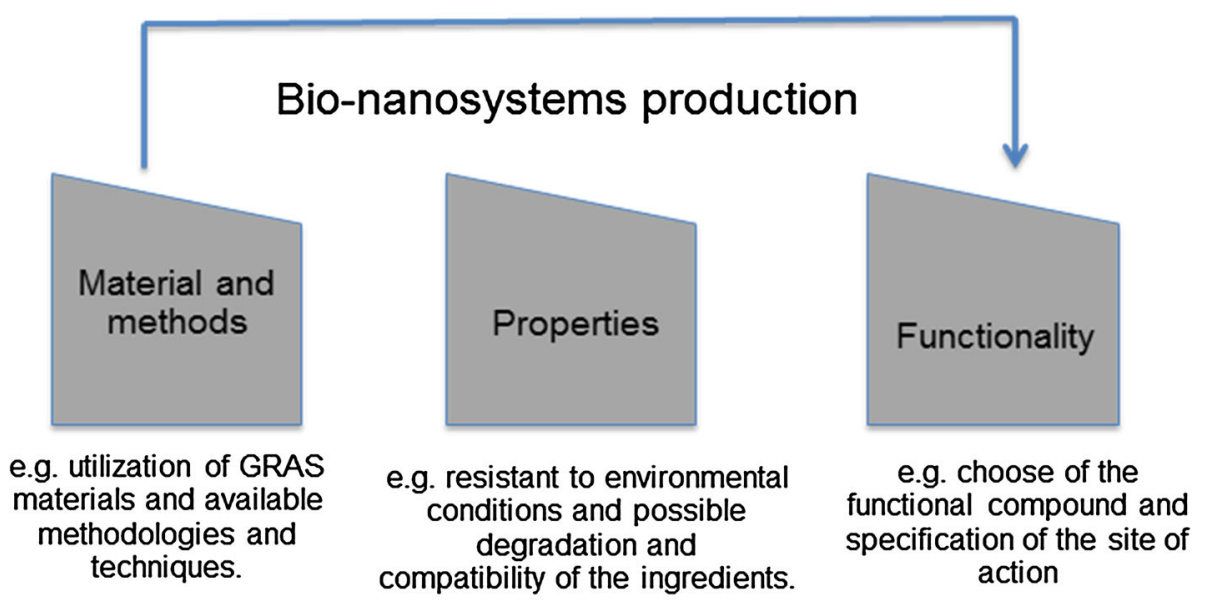

Reverse engineering approach 
in the small intestine and present a high bioavailability; it should also be stable and thus be protected from light and temperature during food processing, storage and digestion (e.g., when passing through the stomach at $\mathrm{pH} \approx 2$ ). Being so, in a reverse engineering approach, the utilization of polysaccharide-based bio-nanosystems will be considered for vitamin B2 encapsulation as they bring advantages since they are not destroyed in acid media and can protect vitamins from light and temperature. Moreover, it may be important that such nanosystems present bioadhesion features (e.g., in order to increase their retention time in the small intestine); in this case, care should be taken to use a polysaccharide with that capacity (e.g., chitosan due to its positive charge can attach to negative compounds existing on epithelial cell membranes) [108]. It should also be taken into account that vitamin B2 is water soluble, which calls for the need of an encapsulation material with hydrophilic behavior (again, e.g., polysaccharides can do that job). Finally, encapsulation of hydrophilic compounds using polysaccharides may be performed using, e.g., ionic pregelation/coacervation. This approach ends with the production and characterization of the nanostructure, verifying that its functionality is in agreement with the initially identified needs.

\section{Future Trends and Consumer Perception}

The development of bio-nanosystems for oral delivery of functional compounds has long been accepted by the scientific community and some industrial players as a possible solution for some of the problems faced by the food industry. However, before nanotechnology being entirely embraced by food industry, there are significant challenges that must be overcome. The use of very small particle sizes may alter the biological fate of the ingested bio-based materials and encapsulated bioactive compounds, which could potentially have adverse effects on human health. Also, the knowledge of the molecular, physicochemical and physiological processes that occur during digestion and absorption of nanosystems will allow the optimized design of nanotechnology-based functional foods for improved health and wellness. Recently, considerable progress has been made in the understanding of the behavior of nanosystems in gastrointestinal tract, however, further work is clearly needed. This knowledge will be crucial to evaluate the biological activity of nanosystems in vivo and to know the potential health risks from their use.

For example, there is currently a lack of understanding of those nanostructures behavior when interacting with human epithelial barriers. The evaluation of these nanosystems using well-differentiated cell lines (e.g., Caco- 2 cell model) could be an effective in vitro model to mimic the characteristics and functions of the epithelium of the small intestine in order to provide a way of addressing issues such as toxicity and permeability. These studies can be a way to guarantee that nanotechnology appears in the next years in food industry as a "safe" technology. It is known that despite the exciting potential of nanotechnology, the scientific community, regulatory authorities and consumers are aware of potential risks that this technology may represent in food products [25]. It is clear that public perception and consumers' attitudes are the major factors determining the commercial success of this field.

A recent study by the Food Safety Agency (US) [109] where consumers were asked about the utilization of nanotechnology in food products showed that when the application of nanotechnology directly to food is at stake, consumers are worried; however, their opinion is more positive if nanotechnology is used in food products with health benefits (where the addition of functional compounds can be included). They were less convinced for other uses of nanotechnology such as in food processing, where changes in food texture or flavor were considered as potential risks not worth to be taken.

A different study about consumer perception of the application of nanotechnology in food products [104] showed that the utilization of nanotechnology in health and nutritional products should be carefully examined due to the relatively low consumer acceptance of these products.

In all the cases, however, it was possible to find consensus in the idea that providing confidence to consumers about the use of nanotechnology in food products implies transparency in the developments achieved by this new area of science and technology, including more information about risks and benefits regarding its utilization in food industry.

Acknowledgments Miguel A. Cerqueira, Ana C. Pinheiro, Hélder D. Silva, Philippe E. Ramos, Ana I. Bourbon, Óscar L. Ramos (SFRH/BPD/72753/2010, SFRH/BD/48120/2008, SFRH/BD/81288/ 2011，SFRH/BD/80800/2011，SFRH/BD/73178/2010 and SFRH/ $\mathrm{BPD} / 80766 / 2011$, respectively) are the recipients of a fellowship from the Fundação para a Ciência e Tecnologia (FCT, POPH-QREN and FSE Portugal). María L. Flores-López thanks Mexican Science and Technology Council (CONACYT, Mexico) for PhD fellowship support (CONACYT Grant number: 215499/310847). The support of EU Cost Actions FA0904 and FA1001 is gratefully acknowledged.

\section{References}

1. Acosta E (2009) Bioavailability of nanoparticles in nutrient and nutraceutical delivery. Curr Opin Colloid Interface Sci 14:3-15

2. Ahmed K, Li Y, McClements DJ, Xiao H (2012) Nanoemulsionand emulsion-based delivery systems for curcumin: encapsulation and release properties. Food Chem 132(2):799-807

3. Angelatos AS, Johnston APR, Wang YJ, Caruso F (2007) Probing the permeability of polyelectrolyte multilayer capsules via a molecular beacon approach. Langmuir 23(8):4554-4562 
4. Aoki T, Decker EA, McClements DJ (2005) Influence of environmental stresses on stability of $\mathrm{O} / \mathrm{W}$ emulsions containing droplets stabilized by multilayered membranes produced by a layer-by-layer electrostatic deposition technique. Food Hydrocoll 19:209-220

5. Arunkumar R, Prashanth KVH, Baskaran V (2013) Promising interaction between nanoencapsulated lutein with low molecular weight chitosan: characterization and bioavailability of lutein in vitro and in vivo. Food Chem 141(1):327-337

6. Barichello JM, Morishita M, Takayama K, Nagai T (1999) Encapsulation of hydrophilic and lipophilic drugs in PLGA nanoparticles by the nanoprecipitation method. Drug Dev Indust Pharm 25(4):471-476

7. Bazylińska U, Warszyński P, Wilk KA (2012) Influence of pH upon in vitro sustained dye-release from oil-core nanocapsules with multilayer shells. Colloid Surf A 413:266-272

8. Bengoechea C, Jones OG, Guerrero A, McClements DJ (2011) Formation and characterization of lactoferrin/pectin electrostatic complexes: impact of composition, $\mathrm{pH}$ and thermal treatment. Food Hydrocoll 25(5):1227-1232

9. Berton-Carabin CC, Coupland JN, Elias RJ (2013) Effect of the lipophilicity of model ingredients on their location and reactivity in emulsions and solid lipid nanoparticles. Colloids Surf A Physicochem Eng Aspects 431:9-17

10. Bertrand P, Jonas A, Laschewsky A, Legras R (2000) Ultrathin polymer coatings by complexation of polyelectrolytes at interfaces: suitable materials, structure and properties. Macromol Rapid Commun 21:319-348

11. Beyer S, Bai J, Blocki A, Kantak C, Xue Q, Raghunath M, Trau D (2012) Assembly of biomacromolecule loaded polyelectrolyte multilayer capsules by using water soluble sacrificial templates. Soft Matter 8:2760-2768

12. Bryant CM, McClements DJ (1998) Molecular basis of protein functionality with special consideration of cold-set gels derived from heat-denatured whey. Trends Food Sci Tech 9(4):143-151

13. Cai S, Yang Q, Bagby TR, Forrest ML (2011) Lymphatic drug delivery using engineered liposomes and solid lipid nanoparticles. Adv Drug Deliv Rev 63(10-11):901-908

14. Calvo P, Remuñán-Lopez C, Vila-Jato JL, Alonso MJ (1997) Novel hydrophilic chitosan-polyethylene oxide nanoparticles as protein carriers. J Appl Polym Sci 63:125-132

15. Chakraborty S, Shukla D, Mishra B, Singh S (2009) Lipid-an emerging platform for oral delivery of drugs with poor bioavailability. Eur J Pharm Biopharm 73(1):1-15

16. Chaudhry Q, Castle L, Watkins R (2010) Nanotechnologies in the food arena: new opportunities, new questions, new concerns. In: Chaudhry Q, Castle L, Watkins R (eds) Nanotechnologies in food. Royal Society of Chemistry, Cambridge, pp 1-17

17. Chen L, Remondetto GE, Subirade M (2006) Food proteinbased materials as nutraceutical delivery systems. Trends Food Sci Tech 17:272-283

18. Chern CS (2006) Emulsion polymerization mechanisms and kinetics. Prog Polym Sci 31:443-486

19. Choi A-J, Kim C-J, Cho Y-J, Hwang J-K, Kim C-T (2011) Characterization of capsaicin-loaded nanoemulsions stabilized with alginate and chitosan by self-assembly. Food Bioprocess Tech 4:1119-1126

20. Cushen M, Kerry J, Morris M, Cruz-Romero M, Cummins E (2012) Nanotechnologies in the food industry - recent developments, risks and regulation. Trends Food Sci Tech 24(1):30-46

21. Das RK, Kasoju N, Bora U (2010) Encapsulation of curcumin in alginate-chitosan-pluronic composite nanoparticles for delivery to cancer cells. Nanomed Nanotechnol 6(1):153-160

22. Das S, Choudhary A (2011) Recent advances in lipid nanoparticle formulations with solid matrix for oral drug delivery. AAPS PharmSci Tech 12:62-76
23. Dickinson E (2003) Colloidal aggregation: mechanism and implications. In: Dickinson E, van Vlie T (eds) Food colloids, biopolymers and materials. Royal Society of Chemistry, Cambridge, pp 68-83

24. de Villiers MM, Otto DP, Strydom SJ, Lvov YM (2011) Introduction to nanocoatings produced by layer-by-layer (LbL) selfassembly. Adv Drug Deliv Rev 63(9):701-715

25. European Commission (2013) Nanotechnology: the invisible giant tackling Europe's future challenges. European Union. http://ec. europa.eu/research/industrial_technologies/pdf/nanotechnology_ en.pdf.

26. Ezhilarasi PN, Karthik P, Chhanwal N, Anandharamakrisshnan C (2013) Nanoencapsulation techniques for food bioactive components: a review. Food Bioprocess Technol 6:628-647

27. Fang Z, Bhandari B (2010) Encapsulation of polyphenols-a review. Trends Food Sci Tech 21(10):510-523

28. Filipcsei G, Csetneki I, Szilágyi A, Zrínyi M (2007) Magnetic field responsive smart polymer composites. Adv Polym Sci 206:137-189

29. Goycoolea FM, Lollo G, Remuñán-López C, Quaglia F, Alonso MJ (2009) Chitosan-alginate blended nanoparticles as carriers for the transmucosal delivery of macromolecules. Biomacromolecules 10:1736-1743

30. Gu YS, Decker EA, McClements DJ (2007) Application of multi-component biopolymer layers to improve the freeze-thaw stability of oil-in-water emulsions: $\beta$-lactoglobulin-1-carrageenan-gelatin. J Food Eng 80(4):1246-1254

31. Gulrez SKH, Saphwan A, Phillips GO (2011) Hydrogels: methods of preparation, characterisation and applications. In: Carpi A (ed) Progress in molecular and environmental bioengineering-from analysis and modeling to technology applications. InTech, Winchester, pp 117-150

32. Guzey D, McClements DJ (2006) Formation, stability and properties of multilayer emulsions for application in the food industry. Adv Colloid Interface 128-130:227-248

33. Gyarmati B, Némethy Á, Szilágyi A (2013) Reversible disulphide formation in polymer networks: a versatile functional group from synthesis to applications. Eur Polym J 49(6):1268-1286

34. Helgason T, Awad TS, Kristbergsson K, McClements DJ, Weiss J (2009) Effect of surfactante surface coverage on formation of solid lipid nanoparticles (SLN). J Colloid Interface Sci 334(1):75-81

35. Hennink WE, Nostrum CFV (2002) Novel crosslinking methods to design hydrogels. Adv Drug Deliv Rev 54:13-36

36. Hoare TR, Kohane DS (2008) Hydrogels in drug delivery: progress and challenges. Polymer 49(8):1993-2007

37. Hoffman AS (2002) Hydrogels for biomedical applications. Adv Drug Deliv Rev 54(1):3-12

38. Hou Z, Gao Y, Yuan F, Liu Y, Li C, Xu D (2010) Investigation into the physicochemical stability and rheological properties of $\beta$-carotene emulsion stabilized by soybean soluble polysaccharides and chitosan. J Agric Food Chem 58(15):8604-8611

39. Hu J, Yu S, Yao P (2007) Stable amphoteric nanogels made of ovalbumin and ovotransferin via self-assembly. Langmuir 23:6358-6364

40. Hu B, Pan C, Sun Y, Hou Z, Ye H, Zeng X (2008) Optimization of fabrication parameters to produce chitosan-tripolyphosphate nanoparticles for delivery of tea catechins. J Agric Food Chem 56(16):7451-7458

41. Huang Q, Yu H, Ru Q (2010) Bioavailability and delivery of nutraceuticals using nanotechnology. J Food Sci 75:R50-R57

42. Jamróz E, Para G, Jachimska B, Szczepanowicz K, Warszyński P, Para A (2013) Albumin-furcellaran complexes as cores for nanoencapsulation. Colloids Surf A. doi:10.1016/j.colsurfa. 2013.01.002 
43. Jo C, Kang H, Lee NY, Kwon JH, Byun MW (2005) Pectin- and gelatin-based film: effect of gamma irradiation on the mechanical properties and biodegradation. Radiat Phys Chem 72(6):745-750

44. Johnston A, Such G, Dodds S, Caruso F (2012) In: Decher G (ed) Engineered layer-by-layer assembled capsules for biomedical applications, 2nd edn. Wiley-VCH Verlag GmbH \& Co. KGaA, New York

45. Kawaguchi S, Ito K (2005) Dispersion polymerization. Adv Polym Sci 175:299-328

46. Kopecek J (2003) Smart and genetically engineered biomaterials and drug delivery systems. Eur J Pharm Sci 20:1-16

47. Lertsutthiwong P, Noomun K, Jongaroonngamsang N, Rojsitthisak P, Nimmannit U (2008) Preparation of alginate nanocapsules containing turmeric oil. Carbohydr Polym 74:209-214

48. Li Y, McClements DJ (2010) New mathematical model for interpreting $\mathrm{pH}$-stat digestion profiles: impact of lipid droplet characteristics on in vitro digestibility. J Agric Food Chem 58(13):8085-8092

49. Li Q, Liu C-H, Huang Z-H, Xue F-F (2011) Preparation and characterization of nanoparticles based on hydrophobic alginate derivative as carriers for sustained release of vitamin D3. J Agric Food Chem 59(5):1962-1967

50. Lin CC, Metters AT (2006) Hydrogels in controlled release formulations: network design and mathematical modeling. Adv Drug Deliv Rev 58:1379-1408

51. Liu F, Urban MW (2010) Recent advances and challenges in designing stimuli-responsive polymers. Prog Polym Sci 35:3-23

52. Loretta L, Rivera-Gil P, Abbasi A, Ochs M, Ganas C, Zins I, Soonnichsen C, Parak W (2010) LbL multilayer capsules: recent progress and future outlook for their use in life sciences. Nanoscale 2:458-467

53. Luo Y, Teng Z, Wang Q (2012) Development of zein nanoparticles coated with carboxymethyl chitosan for encapsulation and controlled release of vitamin D3. J Agric Food Chem 60:836-843

54. Madureira AR, Pereira CI, Gomes AMP, Pintado ME, Malcata FX (2007) Bovine whey proteins-overview on their main biological properties. Food Res Int 40(10):1197-1211

55. Maltais A, Remondetto GE, Gonzalez R, Subirade M (2005) Formation of soy protein isolate cold-set gels: protein and salt effects. J Food Sci 70(1):67-73

56. Mantilla N, Castell-Perez ME, Gomes C, Moreira RG (2013) Multilayered antimicrobial edible coating and its effect on quality and shelf-life of fresh-cut pineapple (Ananas comosus). LWT Food Sci Technol 51:37-43

57. McClements DJ (2012) Advances in fabrication of emulsions with enhanced functionality using structural design principles. Adv Colloid Interface Sci 17(5):235-245

58. McClements DJ, Decker EA, Park Y (2009) Controlling Lipid Bioavailability through Physicochemical and Structural Approaches. Crit Rev Food Sci 49(1):48-67

59. McClements DJ, Decker EA, Weiss J (2007) Emulsion-based delivery systems for lipophilic bioactive components. J Food Sci 72(8):109-124

60. McClements DJ, Xiao H (2012) Potential biological fate of ingested nanoemulsions: influence of particle characteristics. Food Funct 3(3):202-220

61. McClements DJ (2010) Design of nano-laminated coatings to control bioavailability of lipophilic food components. J Food Sci 75(1):R30-R42

62. Medeiros BGS, Pinheiro AC, Teixeira JA, Vicente AA, Carneiro-da-Cunha MG (2012) Polysaccharide/protein nanomultilayer coatings: construction, characterization and evaluation of their effect on 'Rocha' Pear (Pyrus communis L.) shelf-life. Food Bioprocess Tech 5(6):2435-2445
63. Mehnert W, Mader K (2001) Solid lipid nanoparticles-production, characterization and applications. Adv Drug Deliv Rev 47:165-196

64. Mohanraj VJ, Chen Y (2006) Nanoparticles-a review. Trop J Pharm Res 5(1):561-573

65. Mora-Huertas CE, Fessi H, Elaissari A (2010) Polymer-based nanocapsules for drug delivery. Int J Pharm 385:113-142

66. Morris VJ (2010) Natural food nanostructures. In: Chaudhry Q, Castle L, Watkins R (eds) Nanotechnologies in food. Royal Society of Chemistry, Cambridge, pp 50-68

67. Moskowitz H (2000) Inter-relating data sets for product development: the reverse engineering approach. Food Qual Prefer $11: 105-119$

68. Moskowitz HR, Maier A (2007) Response surface methodology and consumer-driven product optimization. In: Beckley JH, Foley MM, Topp EJ, Huang JC, Prinyawiwatkul W (eds) Accelerating new food product design and development. Blackwell Publishing and the Institute of Food Technologists, New York, pp 297-349

69. Mulvihill DM, Donovan M (1987) Whey proteins and their thermal-denaturation-a review. Ir J Food Sci Tech 11:43-75

70. Neethirajan S, Jayas DS (2011) Nanotechnology for the food and bioprocessing industries. Food Bioprocess Technol 4:39-47

71. Negi JS, Chattopadhyay P, Sharma AK, Ram V (2013) Development of solid lipid nanoparticles (SLNs) of lopinavir using hot self nano-emulsification (SNE) technique. Eur J Pharm Biopharm 48(1-2):231-239

72. Noack A, Hause G, Mader K (2012) Physicochemical characterization of curcuminoid-loaded solid lipid nanoparticles. Int $\mathbf{J}$ Pharm 423(2):440-451

73. Ochs CJ, Such GK, Yan Y, van Koeverden MP, Caruso F (2010) Biodegradable click capsules with engineered drug-loaded multilayers. ACS Nano 4(3):1653-1663

74. Oh JK, Lee DI, Park JM (2009) Biopolymer-based microgels/ nanogels for drug delivery applications. Prog Polym Sci 34(12):1261-1282

75. Otto KN, Wood KL (1998) Product evolution: a reverse engineering and redesign methodology. Res Eng Des 10:226-243

76. Öztürk N, Bereli N, Akgöl S, Denizli A (2008) High capacity binding of antibodies by poly(hydroxyethyl methacrylate) nanoparticles. Colloids Surf B Biointerfaces 67:14-19

77. Pal SL, Jana U, Manna PK, Mohanta GP, Manavalan R (2011) Nanoparticle: an overview of preparation and characterization. J Pharm Sci 1(6):228-234

78. Patil JS, Kamalapur MV, Marapur SC, Kadam DV (2010) Ionotropic gelation and polyelectrolyte complexation: the novel techniques to design hydrogel particulate sustained, modulated drug delivery system: a review. Dig J Nanomater Bios 5(1):241-248

79. Peppas NA, Hilt JZ, Khademhosseini A, Langer R (2006) Hydrogels in biology and medicine: from molecular principles to bionanotechnology. Adv Mater 18:1345-1360

80. Pinheiro AC, Bourbon AI, Medeiros BGS, da Silva LHM, da Silva MCH, Carneiro da-Cunha MG, Coimbra MA, Vicente AA (2012) Interactions between $\kappa$-carrageenan and chitosan in nanolayered coatings-Structural and transport properties. Carbohydr Polym 87(2):1081-1090

81. Pinheiro AC, Bourbon AI, Quintas MAC, Coimbra MA, Vicente AA (2012) $\kappa$-carrageenan/chitosan nanolayered coating for controlled release of a model bioactive compound. Innov Food Sci Emerg 16:227-232

82. Pinheiro AC, Lad M, Silva HD, Coimbra MA, Boland M, Vicente AA (2013) Unravelling the behaviour of curcumin nanoemulsions during in vitro digestion: effect of the surface charge. Soft Matter 9(11):3147-3154 
83. Qian C, Decker EA, Xiao H, McClements DJ (2012) Physical and chemical stability of $\beta$-carotene-enriched nanoemulsions: influence of $\mathrm{pH}$, ionic strength, temperature, and emulsifier type. Food Chem 132(3):1221-1229

84. Quintanar-Guerrero D, Allémann E, Doelker E, Fessi H (1998) Preparation and characterization of nanocapsules from performed polymers by a new process based on emulsificationdiffusion technique. Pharm Res 15(7):1056-1062

85. Ramos OL, Pereira JO, Silva SI, Amorim MM, Fernandes JC, Lopes-da-Silva JA, Pintado ME, Malcata FX (2012) Effect of composition of commercial whey protein preparations upon gelation at various pH values. Food Res Int 48:681-689

86. Rao JP, Geckeler KE (2011) Polymer nanoparticles: preparation techniques and size-control parameters. Prog Polym Sci 36:887-913

87. Reis CP, Neufeld RJ, Ribeiro AJ, Veiga F (2006) Nanoencapsulation I. Methods for preparation of drug-loaded polymeric nanoparticles. Nanomed Nanotechnol 2(1):8-21

88. Rubinstein M, Colby RH (2003) Polymer physics. Oxford University Press, Oxford

89. Said HM, Alla SGA, El-Naggar AWM (2004) Synthesis and characterization of novel gels based on carboxymethyl cellulose/ acrylic acid prepared by electron beam irradiation. React Funct Polym 61:397-404

90. Salvia-Trujillo L, Qian C, Martín-Belloso O, McClements DJ (2013) Influence of particle size on lipid digestion and $\beta$-carotene bioaccessibility in emulsions and nanoemulsions. Food Chem 141(2):1472-1480

91. Sanguansri P, Augustin MA (2006) Nanoscale materials development-a food industry perspective. Trends Food Sci Tech 17:547-556

92. Sarmento B, Ribeiro AJ, Veiga F, Ferreira DC, Neufeld RJ (2007) Insulin-loaded nanoparticles are prepared by alginate ionotropic pre-gelation followed by chitosan polyelectrolyte complexation. J Nanosci Nanotechnol 7:1-9

93. Schmitt C, Bovay C, Vuilliomenet A-M, Rouvet M, Bovetto L, Barbar R, Sanchez C (2009) Multiscale characterization of individualized $\beta$-lactoglobulin microgels formed upon heat treatment under narrow $\mathrm{pH}$ range conditions. Langmuir 25(14):7899-7909

94. Schuetz YB, Gurny R, Jordan O (2008) A novel thermoresponsive hydrogel based on chitosan. Eur J Pharmac Biopharm 68:19-25

95. Sessa M, Casazza A, Perego P, Tsao R, Ferrari G, Donsí F (2013) Exploitation of polyphenolic extracts from grape marc as natural antioxidants by encapsulation in lipid-based nanodelivery systems. Food Bioprocess Tech 6(10):2609-2620

96. Sessa M, Tsao R, Liu R, Ferrari G, Donsí F (2011) Evaluation of the stability and antioxidant activity of nanoencapsulated resveratrol during in vitro digestion. J Agric Food Chem 59(23):12352-12360

97. Shakeel F, Baboota S, Ahuja A, Ali J, Aqil M, Shafiq S (2007) Nanoemulsions as vehicles for transdermal delivery of aceclofenac. AAPS Pharm Sci Tech 8:E104

98. Shiga $T$ (1997) Deformation and viscoelastic behavior of polymer gels in electric fields. Adv Polym Sci 134:131-163

99. Shrivastava S, Dash D (2012) Nanotechnology in food sector and agriculture. Proc Natl A Sci India B 82:29-35

100. Shu S, Sun C, Zhang X, Wu Z, Wang Z, Li C (2010) Hollow and degradable polyelectrolyte nanocapsules for protein drug delivery. Acta Biomater 6:210-217

101. Sneharani AH, Karakkat JV, Singh SA, Rao AGA (2010) Interaction of curcumin with $\beta$-lactoglobulin-stability, spectroscopic analysis, and molecular modeling of the complex. J Agric Food Chem 58(20):11130-11139
102. Silva HD, Cerqueira MA, Vicente AA (2012) Nanoemulsions for food applications: development and characterization. Food Bioprocess Tech 5(3):854-867

103. Silva HD, Cerqueira MA, Souza BWS, Ribeiro C, Avides MC, Quintas MAC, Coimbra JSR, Carneiro-da-Cunha MG, Vicente AA (2011) Nanoemulsions of [beta]-carotene using a highenergy emulsification-evaporation technique. J Food Eng 102(2):130-135

104. Siegrist M, Stampfli N, Kastenholz H, Keller C (2008) Perceived risks and perceived benefits of different nanotechnology foods and nanotechnology food packaging. Appetite 51:283-290

105. Somchue W, Sermsri W, Shiowatana J, Siripinyanond A (2009) Encapsulation of $\alpha$-tocopherol in protein-based delivery particles. Food Res Int 42(8):909-914

106. Soppimath KS, Aminabhavi TM, Kulkarni AR, Rudzinski WE (2001) Biodegradable polymeric nanoparticles as drug delivery devices. J Control Release 70:1-20

107. Spigno G, Donsì F, Amendola D, Sessa M, Ferrari G, De Faveri DM (2013) Nanoencapsulation systems to improve solubility and antioxidant efficiency of a grape marc extract into hazelnut paste. J Food Eng 114(2):207-214

108. Shukla SK, Mishra AK, Arotiba OA, Mamba BB (2013) Chitosan-based nanomaterials: a state-of-the-art review. Int $\mathrm{J}$ Biol Macromol 59:46-55

109. TNS-BMRB Report (2011) FSA citizens' forums: nanotechnology and food. 2011. JN 219186, April

110. Tokarev I, Minko S (2009) Stimuli-responsive hydrogel thin films. Soft Matter 5:511-524

111. Tokle T, Lesmes U, McClements DJ (2010) Impact of electrostatic deposition of anionic polysaccharides on the stability of oil droplets coated by lactoferrin. J Agric Food Chem 58(17):9825-9832

112. Totosaus A, Montejano J, Salazar J, Guerrero I (2002) A review of physical and chemical protein gel induction. Int J Food Sci Tech 37:589-601

113. Ubbink J, Krüger J (2006) Physical approaches for the delivery of active ingredients in Foods. Trends Food Sci Tech 17:244-254

114. Van Boekel MAJS (2005) Technological innovation in food industry: product design. In: Jongen WMF, Meulenberg MTG (eds) Innovation in agri-food systems: product quality and consumer acceptance. Wageningen Academic Publishers, Wageningen, pp 147-172

115. Vermonden T, Censi R, Hennink WE (2012) Hydrogels for protein delivery. Chem Rev 112:2853-2888

116. Wang Y, Angelatos A, Caruso F (2007) Template synthesis of nanostructured materials via layer-by-layer assembly. Chem Mater 20:848-858

117. Wang JC, Chen SH, Xu ZC (2008) Synthesis and properties research on the nanocapsulated capsaicin by simple coacervation method. J Dispers Sci Technol 29(5):687-695

118. Wang Y, Padua GW (2012) Nanoscale characterization of zein self-assembly. Langmuir 28:2429-2435

119. Weiss J, Takhistov P, McClements DJ (2006) Functional materials in food nanotechnology. J Food Sci 71:R107-R116

120. Wong HL, Bendayan R, Rauth AM, Li Y, Wu XY (2007) Chemotherapy with anticancer drugs encapsulated in solid lipid nanoparticles. Adv Drug Deliv Rev 59(6):491-504

121. Ye S, Wang C, Liu X, Tong Z (2005) Multilayer nanocapsules of polysaccharide chitosan and alginate through layer-by-layer assembly directly on PS nanoparticles for release. J Biomater Sci Polym 16:909-923

122. Yu S, Yao P, Jiang M, Zhang G (2006) Nanogels prepared by self-assembly of oppositely charged globular proteins. Biopolymers $83: 148-158$ 
123. Yuxi L, Jing Y, Ziqi Z, Junjie L, Rui Z, Fanglian Y (2012) Formation and characterization of natural polysaccharide hollow nanocapsules via template layer-by-layer self-assembly. J Colloid Interface Sci 379:130-140

124. Zhang F-A, Yu C-L (2007) Acrylic emulsifier-free emulsion polymerization containing hydrophilic hydroxyl monomer in the presence or absence of nano- $\mathrm{SiO}_{2}$. Eur Polym J 43:1105-1111

125. Zhao C, Zhuang X, He P, Xiao C, He C, Sun J, Chen X, Jing X (2009) Synthesis of biodegradable thermo- and $\mathrm{pH}$-responsive hydrogels for controlled drug release. Polymer 50:4308-4316
126. Ziegler GR, Foegeding EA (1990) The gelation of proteins. Adv Food Nutr Res 34:203-298

127. Zimet P, Livney YD (2009) $\beta$-Lactoglobulin and its nanocomplexes with pectin as vehicles for $\omega-3$ polyunsaturated fatty acids. Food Hydrocoll 23(4):1120-1126 